Pak. j. sci. ind. res. Ser. B: biol. sci. 2021 64B(3) 288-300

\title{
A Survey Report of Disease Fields of District; Swabi, Buner, Haripur and Mardan Province of Khyber Pakhtunkhwa
}

\author{
Gulnaz Parveen ${ }^{a *}$, Salma Gul ${ }^{\mathrm{b}}$, Kaleemullah', Zubia Rahima and M.Ather Rafid \\ ${ }^{a}$ Department of Botany, Women University, Swabi, KP, Pakistan \\ ${ }^{b}$ Department of Chemistry, Women University, Swabi, KP, Pakistan \\ ${ }^{c}$ Department of Statistics, Women University, Swabi, KP, Pakistan \\ ${ }^{\mathrm{d} D e p a r t m e n t ~ o f ~ Z o o l o g y, ~ W o m e n ~ U n i v e r s i t y, ~ S w a b i, ~ K P, ~ P a k i s t a n ~}$
}

(received July 15, 2019; revised December 3, 2019; accepted January 1, 2020)

\begin{abstract}
A survey of diseased fields of total (35) areas located in four different Districts of Khyber Pakhtun Khwa like Buner (4 areas), Swabi (23 areas) Mardan (3 areas) and Haripur (5areas) was carried out for estimation of losses and identification of root disease causing pathogens in 14 different economically important crops like Tobacco (Nicotiana tabacum), Ladyfinger (Abelmoscus esculentus), Chilli (Capsicum anuum), Eggplant (Solanum melongena), Maize (Zea mays), Tomato (Solanum lycopersicum), Wheat (Triticum estivum), Garlic (Allium sativum), Mustard (Brassica compestres), Clover (Trifolium repens), Onion (Allium cepa), Turnip (Brassica rapa), Radish (Raphanus sativus) and Pea (Pisum sativum). The most common root rot pathogens found in all the areas visited were Alternaria alternate, A.solani, Rhizoctonia solani Macrophomina phaseolina and species of Fusarium viz., Fusarium solani and Foxysporum. Two species of root-knot nematode Meloidogyne javanica and M. incognita were also found causing rootknot disease. The eggplant was found upto $20-76 \%$ losses when an infection caused by the combined effect of F. oxysporum, M.phaseolina. R. solani and root-knot nematodes. Similarly, the loss of chiliplants was found $40-65 \%$ due to the combined effect of $F$. solani, M.phaseolina, R. solani, and root-knot nematodes. Zea mays crops were reported $50-77 \%$ losses due to Drecslera spp, F.solani, and A. solani Losses recorded by these root-rot and root-knot pathogens were found variable depending upon a combination of pathogens, temperature and soil types.
\end{abstract}

Keywords: colonization, infection, pathogens, root-rot, swabi

\section{Introduction}

Agriculture is the backbone of Pakistan's economy on which the population of the country is dependent and also accounts for $26 \%$ ofthe productivity of the country. Cotton, rice, wheat, sugarcane, vegetables, and fruits are the major crops of Pakistan (Rehman et al., 2015). Pathogenic organisms damage vegetable crops and reduce production by killing the plants thus making the products unmarketable (Shafique et al., 2016).

Losses of crops highly threaten the rural farmer's income as well as the food productivity of the world. Pests and diseases are the major cause of such losses (Avelino et al., 2015). Crop losses areavery remarkable problem in Pakistan and all over the world. Plant-parasitic nematodes caused losses of US\$ 100 billion per year to world agriculture (Kayani et al., 2018; Tariq-Khan et al., 2017).

Losses caused by pathogens, pests, insects, bacteria, fungi, and viruses are favoured by poor infrastructure,

*Author for correspondence;

E-mail: gulnaz.malik3@gmail.com
Post-harvest handling and climate change. A mycotoxin is also a problem creator. Fungi releases mycotoxins which are continuously contaminating the maize crops in store houses as well as in fields. Mismanagement of mycotoxin contamination of maize affects humans as well as animal's health (Suleiman and Kurt, 2015).

The soil-borne plant pathogens infecting plant roots are one of the most important problems facing world agriculture that reduces yield and quality in economic crops (Aslam et al., 2017 a,b; Katan, 2017). In Pakistan, the pathogenic fungi and nematodes attacked different crops (Zarins and Shahina, 2010). The loss of vegetables in quantity and quality is due to their susceptibility to many soil and root diseases (Chehri et al., 2010). Among the most important plant pathogens, Fusarium and its most of the species recognized as major cause of root infection, reported in Sindh and Balochistan (Parveen et al., 2020), it causes great losses in grain crops globally (Savary et al., 2012).

Wheat, Rice, and Maize are the most demanding crops of the world (Suleiman et al., 2013). Almost 10.14 
billion metric tons of maize is currently producing worldwide (De Groote et al., 2013). A huge amount of (grain) maize is stored and harvested under humid and hot climate in tropics and subtropics because hot and humid climate conditions facilitates fungal growth of maize (Egal et al., 2005), foremost lack of knowledge of equipment and drying methods also lead to wastage (Weinberg et al., 2008)

Pakistan is facing anannual loss of eggplant (Solanum melongena L.) due to its high susceptibility to root rotting fungi F. oxysporum, Macrophomina phaseolina, Fusarium solani and root-knot nematode (Meloidogyne spp.) (Baloch et al., 2013). Almost 500 species of more than 100 families of plants are highly susceptible to $M$. phaseolina which include food source (maize and sorghum), vegetables (tomato, potato, onion and garlic) oil producers (sunflower, soybean and castor), pulses (green gram, mung bean, groundnut and sesame) and fiber producers (cotton) (Das et al., 2008). Infection caused to these crops remains in the soil for more than 4 years in the form of sclerotia (Islam et al., 2012). At the onset of favourable conditions, sclerotia penetrate the cell wall by producing hyphae and cause disease (Suleiman and Kurt, 2015).

In Pakistan, different plant species like Mango (Mohsan et al., 2011), Tomato (Akhtar et al., 2004) and Aloe vera (Bajwa et al., 2010) are affected by common disease caused bya black spot of Alternaria. Major plant pathogens are Alternaria species cause $20 \%$ losses and most serve the losses reach up to $80 \%$. The leaves, stems, flowers and fruits are affecting by these pathogens (Nowicki et al., 2012), while 38.8\% loss was reported of Okra in the area of Punjab (Husain et al., 2012). Losses ranged $(6.75 \%$ to $15.5 \%)$ and root incidence ranged were estimated by root rot fungi in Multan. (Inam-ul-Haque et al., 2012).

Remarkable research on losses by soil-borne plant pathogens has not been done in Pakistan. The existing report, therefore, describes the estimation of losses caused by root rotting fungi and root-knot nematodes in some important crops grown in Khyber Pakhtunkhwa.

Survey of diseased fields for the assessment of crop losses. A survey of diseased fields of total (35) areas located in four different districts of Khyber Pakhtunkhwa like Buner including 4 different areas: (Nogram, Panjtar, Chengli and Buner), Swabi including 23 different locations (Seen Khel, Gohati, Bikot, Bamkhel, Saleem Khan, Maneri, Bikot, Yarhussain, Manki, Shahid Banda,
Ismaila, Topi, Sudher, Dagai, Tordhor, Kernnal Sher khan, Dobian, Marghuz, Jalabai, Darra, Maini, Kunda, Shahmansoor and Kalu Khan) Mardan (Shahbaz Gallei, Takhat Baye and Sheik meltoon) and Haripur included 5 locations (Ghazi, Haripure, Bagh banda, Nelor, Parhala) was carried out for estimation of losses in different seasons due to root-knot nematodes and root infecting fungi in 14 different economically important crops. An interview was conducted from farmers to analyze the estimated production and actual production which was recorded.

Specimens of roots of plants infected with root-knot and root-rot pathogens were collected and examined in the laboratory for identification and isolation of organisms causing disease. From each field, five samples were collected which were then kept at $4{ }^{\circ} \mathrm{C}$ and the isolation has been done within $24 \mathrm{~h}$.

Determination of crop losses percentage. The percentage of crop losses by root infecting fungi was determined by the following formula.

Losses $\%=\frac{\text { Obtained yield (diseased plants) }}{\text { Standard yield of healthy plants }} \times 100$

Isolation of fungi from roots. Root samples were washed under tap water and $1 \% \mathrm{Ca}(\mathrm{OCl})_{2}$ was used for surface sterilization. These samples were then transferred on PDA containing Streptomycin $(0.2 \mathrm{~g} / \mathrm{L})$ and penicillin (100000 units/L). Infection and colonization were confirmed after incubation of dishes for a time of 5 days at $28^{\circ} \mathrm{C}$.

Percentage of infection and colonization was calculated as follows:

Infection $\%=\frac{\text { No. of plant infected by pathogen }}{\text { Total no. of plants }} \times 100$

No. of root samples colonized

$\begin{aligned} & \text { Colonization } \%= \frac{\text { by a pathogen }}{\text { Total no. of root sample of }} \\ & \text { all plants }\end{aligned}$

Identification of root-knot nematodes. For this purpose root showing infection was sliced into pieces and dissected using a stereo-microscope. Ten pieces from each sample showing pear-shaped females were collected and transferred to the slide, while samples with the 
perennial pattern were cut from each female using a sharp razor in a dissecting microscope and after comparison with a known pattern of certain nematodes Meloidogyne species were identified after comparing with pattern provided according to Taylor and Sasser (1978).

Root-knot index (0-5 scale). Root-knot index: Infection of roots by knot forming nematode was estimated using a 0-5 scale described by Taylor and Sasser (1978).

Statistical analysis. Data has been analyzed statistically by finding out the Pearson Correlation of infection and colonization percentage and comparison of means losses of different economically important crops by using IBM SPSS STATISTICS (Sokal and Rohlf, 1995).

\section{Results and Discussion}

Estimation of crop losses with association of soilborne plant pathogens in diseased filed. During the survey, fourteen different plant species were taken under consideration to check the losses percentage caused by soil-borne fungus and root-knot nematodes in different areas of Khyber Pakhtunkhawa.

Losses percentage varies among different areas from $10 \%$ to $77 \%$ (Table 1). 25 different pathogenic fungi were found responsible to causes losses in 14 different hosts, while maximum host was infected by M. phaseolina (8 hosts), Alternaria alternata (11 hosts), Fusarium solani (9 hosts), Rhizoctonia solani (10 hosts) Penecilium spp ( 8 hosts) and A. solani (8 hosts) (Table 2).

Among root-knot nematodes Meloidogyne javanica 4(R.K) and M. incognita 3(R.K) both were associated with chili and eggplant (Fig. 1).The maximum infection percentageof $M$. phaseolina $(100 \%)$ was found associated with diseased roots of Nicotiana tabacum, while Pearson correlation (0.924) shown significant at $\mathrm{P}$-value $=0.001$ between percent infection and colonization which was responsible to cause 20 to $52 \%$ losses. The ladyfinger was associated with different pathogens, while infection percentage of $F$. solani was maximum $100 \%$ and Pearson correlation $(0.765)$ revealed significant at $\mathrm{P}$-value $=0.001$ between percent infection and colonization which was and responsible 50 to $72 \%$ losses while mean differences of losses, (58) were found highly significant at $\mathrm{P}$-value $=0.001$. Highest percent infection of M. phaseolina $(100 \%)$ in chili and Pearson Correlation (0.766) shown significant at Pvalue $=0.001$ between percent infection and colonization which caused 25 to $65 \%$ losses and mean differences of losses (50.5) was found highly significant at P-value $=0.001$. The maximum infection percentage of $R$. solan $i$ (100) and F. oxyporum (100) in eggplant and Pearson Correlation $(0.78)$ revealed significant at $\mathrm{P}$-value $=0.001$ between percent infection and colonization which was responsible to cause 20 to $76 \%$ losses, while mean differences of losses (47) was found highly significant at $\mathrm{P}$-value $=0.001$. The Zea maize was associated with different pathogens, while infection percentage of Drecslera spp was maximum $100 \%$ and Pearson Correlation (0.977) reveal significant at $\mathrm{P}$-value $=0.01$ between percent infection and colonization which was responsible 50 to $77 \%$ losses, while mean differences of losses (61.33) were found highly significant at Pvalue $=0.05$. Highest percent infection of $A$. alternata, $R$. solani and $F$. solani were (80\%) in Tomato plant and Pearson Correlation (0.779) represented significant at $\mathrm{P}$-value $=0.05$ between percent infection and colonization which was to cause 14 to $62.5 \%$ losses. The wheat was associated with $A$. alternata was maximum $100 \%$ and Pearson Correlation (0.614) reveal significant at Pvalue $=0.001$ between percent infection and colonization which was responsible 10 to $65 \%$ losses, while mean differences of losses (28.75) was found the highly significant at $\mathrm{P}$-value $=0.001$. The highest percent infection of $A$. solani was ( $80 \%)$ in garlic plant to cause up $60 \%$ losses in Topi, while mean differences of losses (22.5) were found significant at $\mathrm{P}$-value $=0.05$. The maximum infection percentage of $A$. alternata $(80 \%)$, Pearson correlation (0.737) reveal significant at Pvalue $=0.01$ between percent infection and colonization which was found associated with diseased roots of mustard and responsible to cause 15 to $40 \%$ losses, while mean differences of losses (28.29) was found highly significant at $\mathrm{P}$-value $=0.001$. The clover was associated with different pathogens, while infection percentage of M. phaseolina wasa maximum $80 \%$ and responsible up to $48 \%$ losses in Topi, while mean differences of losses (33.25) was found significant at $\mathrm{P}$-value $=0.01$. In onion infection percentage of $F$. oxysporum (80) and Pearson Correlation (0.647) reveal significant at $\mathrm{P}$-value $=0.01$ between percent infection and colonization which was responsible 20 to $60 \%$ losses, while mean differences of losses (27.63) were found highly significant at $\mathrm{P}$-value $=0.001$. The Turnip was associated with different pathogens, while the infection percentage of A. alternata was a maximum of $80 \%$ and responsible up to $80 \%$ losses in Jalabi, while mean differences of losses (46.5) were found 
Table 1. Losses caused by soil borne pathogens in some economic crops in different districts of Khyber Pakhtun Khwa.

\begin{tabular}{|c|c|c|c|c|c|}
\hline Names of crops & Location & $\begin{array}{l}\text { Area of cultivation } \\
\mathrm{Sq} \mathrm{ft}\end{array}$ & $\begin{array}{l}\text { Obtained yeild } \\
(\mathrm{Kg})\end{array}$ & $\begin{array}{l}\text { Standard yeild } \\
(\mathrm{Kg})\end{array}$ & $\begin{array}{l}\text { Losses } \\
\%\end{array}$ \\
\hline \multirow{3}{*}{$\begin{array}{l}\text { Nicotiana tabacum } \\
\text { (Tobacco) }\end{array}$} & Panjtar & 16335 & 400 & 500 & 20 \\
\hline & Seen khel & 81675 & 1000 & 2100 & 52 \\
\hline & Gohati & 544.5 & 90 & 180 & 50 \\
\hline \multirow{6}{*}{$\begin{array}{l}\text { Abelmoscus esculantus } \\
\text { (Ladyfinger) }\end{array}$} & Seen Khel & 544.5 & 85 & 170 & 50 \\
\hline & Gohati & 544.5 & 90 & 180 & 50 \\
\hline & Nogram & 10890 & 600 & 1500 & 66 \\
\hline & Bamkhel & 544.5 & 50 & 180 & 72 \\
\hline & SaleemKhan & 816.75 & 90 & 230 & 60 \\
\hline & Bikot & 38115 & 2500 & 5000 & 50 \\
\hline \multirow{8}{*}{$\begin{array}{l}\text { Capsicum annum } \\
\text { (Chili) }\end{array}$} & Panjtar & 544.5 & 98 & 150 & 65 \\
\hline & Gohati & 272.25 & 4 & 10 & 60 \\
\hline & Nogram & 544.5 & 13 & 25 & 48 \\
\hline & Bamkhel & 272.2 & 3 & 7 & 57 \\
\hline & Saleemkhan & 544.5 & 7 & 20 & 65 \\
\hline & Maneri & 1089 & 30 & 50 & 40 \\
\hline & Kalu khan & 5445 & 50 & 90 & 44 \\
\hline & Yar hussain & 16335 & 600 & 800 & 25 \\
\hline \multirow{10}{*}{$\begin{array}{l}\text { Solanum melongena } \\
\text { (EggPlant) }\end{array}$} & Nogram & 10890 & 60 & 250 & 76 \\
\hline & Bamkhel & 816.75 & 12 & 25 & 52 \\
\hline & Saleemkhan & 10890 & 120 & 250 & 52 \\
\hline & Maneri & 16335 & 100 & 280 & 64 \\
\hline & Kalu khan & 10890 & 200 & 250 & 20 \\
\hline & Maneri & 21780 & 280 & 400 & 30 \\
\hline & Kalu khan & 2722.5 & 17 & 60 & 71 \\
\hline & Gohati & 816.75 & 15 & 20 & 25 \\
\hline & Seen khel & 544.5 & 9 & 18 & 50 \\
\hline & Manki & 5445 & 700 & 1000 & 30 \\
\hline \multirow{3}{*}{$\begin{array}{l}\text { Zea mays } \\
\text { (maize) }\end{array}$} & Seen khel & 43560 & 550 & 2400 & 77 \\
\hline & Bikot & 16335 & 300 & 700 & 57 \\
\hline & Gohati & 1905.75 & 100 & 200 & 50 \\
\hline \multirow{12}{*}{$\begin{array}{l}\text { Solanum lycopersicum } \\
\text { (tomato) }\end{array}$} & Panjtar & 10890 & 200 & 500 & 60 \\
\hline & Shaheed banda & 544.5 & 150 & 400 & 62.5 \\
\hline & Ismaila & 10890 & 3000 & 3500 & 14 \\
\hline & Shahbaz gallei & 27225 & 2300 & 2700 & 15 \\
\hline & Yarhussain & 5445 & 2000 & 2500 & 20 \\
\hline & Parhala & 5445 & 2700 & 3500 & 23 \\
\hline & & 54450 & 4400 & 5000 & 12 \\
\hline & Sudher & 5445 & 2200 & 2500 & 12 \\
\hline & Nelor & 10890 & 1500 & 2000 & 25 \\
\hline & & 21780 & 1500 & 3000 & 50 \\
\hline & Dagai & 16335 & 1200 & 1500 & 20 \\
\hline & Tordher & 10890 & 700 & 1200 & 42 \\
\hline \multirow{9}{*}{$\begin{array}{l}\text { Triticum estivum } \\
\text { (Wheat) }\end{array}$} & & 16335 & 2000 & 2500 & 20 \\
\hline & & 27225 & 1000 & 2000 & 50 \\
\hline & Kernal Khan Sher & 87120 & 1400 & 4000 & 65 \\
\hline & & 87120 & 2800 & 4000 & 40 \\
\hline & Dobian & 43560 & 1200 & 2000 & 40 \\
\hline & Marghuz (2 fields) & 43560 & 800 & 1000 & 20 \\
\hline & & 10890 & 600 & 1000 & 40 \\
\hline & Jalbai & 5445 & 2000 & 3500 & 43 \\
\hline & Darra & 5445 & 3000 & 4000 & 25 \\
\hline
\end{tabular}




\begin{tabular}{|c|c|c|c|c|c|}
\hline & Buner & 27225 & 2800 & 3300 & 15 \\
\hline & Maini & 54450 & 27000 & 30000 & 10 \\
\hline & Kunda & 38115 & 2100 & 2400 & 13 \\
\hline \multirow{6}{*}{$\begin{array}{l}\text { Allium sativum } \\
\text { (Garlic) }\end{array}$} & Darra & 54450 & 2700 & 3000 & 10 \\
\hline & Ghazi & 816.75 & 5 & 6 & 17 \\
\hline & Takhat Baye & 5445 & 4 & 5 & 20 \\
\hline & Kunda & 2178 & 17 & 20 & 15 \\
\hline & Tordher & 27225 & 1750 & 2000 & 12.5 \\
\hline & Topi & 861.7 & 200 & 500 & 60 \\
\hline \multirow{7}{*}{$\begin{array}{l}\text { Brassica compestres } \\
\text { (Mustard) }\end{array}$} & Dobian & 21780 & 1500 & 2000 & 25 \\
\hline & Kalu khan & 27225 & 1200 & 2000 & 40 \\
\hline & Takhat Baye & 3267 & 28 & 40 & 30 \\
\hline & Shahabaz galei & 54450 & 2300 & 2700 & 15 \\
\hline & Bagh banda & & & & \\
\hline & (Haripur) & 8167.5 & 50 & 80 & 38 \\
\hline & Kunda & 816.7 & 8 & 10 & 20 \\
\hline \multirow{4}{*}{$\begin{array}{l}\text { Trifolium repens } \\
\text { (Clover) }\end{array}$} & Swabi & 5445 & 8 & 15 & 47 \\
\hline & Sudher & 5445 & 10 & 15 & 33 \\
\hline & Shaikh meltoon & 5445 & 12 & 20 & 20 \\
\hline & Jalabai & 5445 & 20 & 30 & 33 \\
\hline \multirow{6}{*}{$\begin{array}{l}\text { Allium cepa } \\
\text { (Onion) }\end{array}$} & Thorder & 3267.0 & 120 & 200 & 40 \\
\hline & Chengli & 5445 & 300 & 500 & 40 \\
\hline & Yarhussain & 5445 & 400 & 500 & 20 \\
\hline & & 10890 & 600 & 900 & 25 \\
\hline & Darra & 5445 & 350 & 500 & 30 \\
\hline & Haripur & 10890 & 400 & 1000 & 60 \\
\hline \multirow{5}{*}{$\begin{array}{l}\text { Brassica rapa } \\
\text { (Turnip) }\end{array}$} & Ghazi & 21780 & 2500 & 3500 & 29 \\
\hline & Jalabai & 5445 & 200 & 1000 & 80 \\
\hline & Swabi & 1780 & 200 & 700 & 67 \\
\hline & Sheikh meltoon & 1089 & 50 & 80 & 37 \\
\hline & Shamansoor & 1361.25 & 70 & 95 & 26 \\
\hline \multirow{4}{*}{$\begin{array}{l}\text { Raphanus sativus } \\
\text { (Radish) }\end{array}$} & Thorder & 8167.5 & 700 & 1000 & 30 \\
\hline & Ghazi & 1089 & 70 & 88 & 23 \\
\hline & Kunda & 544.5 & 35 & 45 & 22 \\
\hline & Haripur & 27225 & 2000 & 2500 & 20 \\
\hline (Pisum sativum) & Miani & 10890 & 800 & 1400 & 43 \\
\hline Pea & Jalabai & 5445 & 150 & 400 & 62.5 \\
\hline
\end{tabular}

Table 2. Root-rot and root-knot pathogens associated with some economic crops.

\begin{tabular}{|c|c|c|c|c|}
\hline Names of crops & Location & $\begin{array}{l}\text { Major pathogen } \\
\text { associated with roots }\end{array}$ & Infection $\%$ & Colonization $\%$ \\
\hline $\begin{array}{l}\text { Nicotiana tabacum } \\
\text { (tobacco) }\end{array}$ & $\begin{array}{l}\text { Panjtar } \\
\text { Seen Khel } \\
\text { Gohati }\end{array}$ & $\begin{array}{l}\text { Macrophomina phaseolina } \\
\text { Alternaria alternate } \\
\text { Fusarium solani } \\
\text { M. phaseolina } \\
\text { F. solani } \\
\text { M. phaseolina } \\
\text { F. solani } \\
\text { Rhizoctonia solani }\end{array}$ & $\begin{array}{l}100 \\
20 \\
20 \\
60 \\
20 \\
60 \\
20 \\
60\end{array}$ & $\begin{array}{l}40 \\
4 \\
4 \\
12 \\
4 \\
32 \\
4 \\
32\end{array}$ \\
\hline $\begin{array}{l}\text { Abelmoscus esculantus } \\
\text { (ladyfinger) }\end{array}$ & $\begin{array}{l}\text { Seen Khel } \\
\text { Gohati }\end{array}$ & $\begin{array}{l}\text { M.phaseolina } \\
\text { M. phaseolina } \\
\text { R. solani } \\
\text { Drecslera. spp } \\
\text { R. solani }\end{array}$ & $\begin{array}{l}100 \\
100 \\
60 \\
20 \\
40\end{array}$ & $\begin{array}{l}40 \\
31 \\
13 \\
8 \\
16\end{array}$ \\
\hline
\end{tabular}




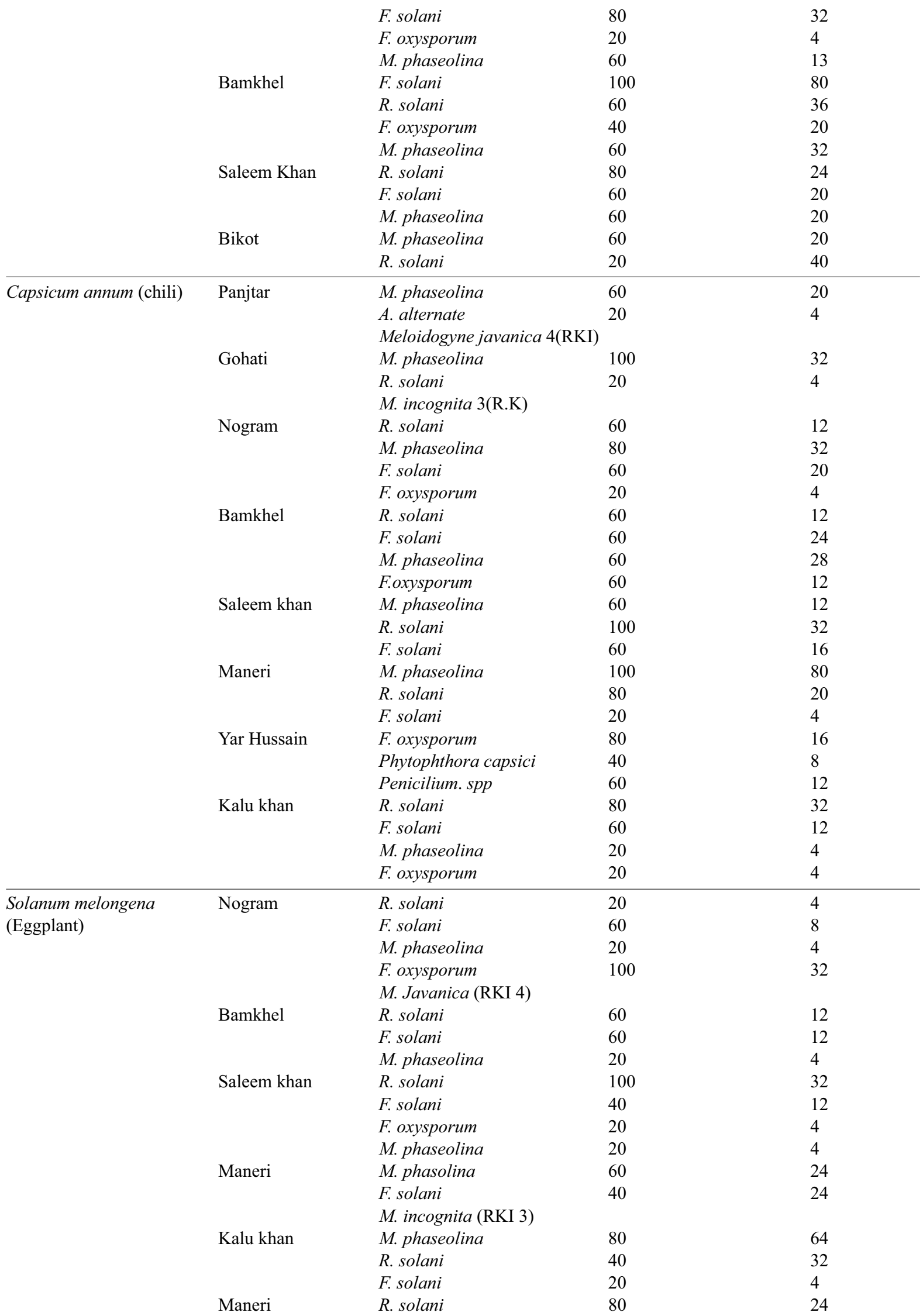




\begin{tabular}{|c|c|c|c|c|}
\hline & & F. oxysporum & 20 & 4 \\
\hline & \multirow[t]{2}{*}{ Kalu khan } & M. phaseolina & 80 & 36 \\
\hline & & F. oxysporum & 20 & 4 \\
\hline & \multirow[t]{2}{*}{ Gohati } & M. phaseolina & 100 & 80 \\
\hline & & F. solani & 60 & 32 \\
\hline & \multirow[t]{2}{*}{ Seen khel } & M. phasiolina & 60 & 32 \\
\hline & & F. solani & 20 & 4 \\
\hline \multirow[t]{5}{*}{ Zea mays (Maize) } & \multirow[t]{3}{*}{ Seen khel } & A. solani & 20 & 4 \\
\hline & & F. solani & 20 & 4 \\
\hline & & Drecslera. spp & 100 & 32 \\
\hline & Bikot & R. solani & 20 & 4 \\
\hline & Gohati & Drecslera. spp & 60 & 12 \\
\hline \multirow{9}{*}{$\begin{array}{l}\text { Solanum lycopersicum } \\
\text { (Tomato) }\end{array}$} & \multirow{3}{*}{ Panjtar } & M. phasiolina & 60 & 12 \\
\hline & & A. alternata & 80 & 32 \\
\hline & & R. solani & 20 & 4 \\
\hline & \multirow[t]{3}{*}{ Shaheed banda } & M. phasiolina & 60 & 32 \\
\hline & & A. alternata & 20 & 4 \\
\hline & & R. solani & 80 & 64 \\
\hline & \multirow[t]{3}{*}{ Ismaila } & F. solani & 80 & 32 \\
\hline & & A. solani & 80 & 32 \\
\hline & & Erysiphe. spp & 60 & 16 \\
\hline \multirow[t]{37}{*}{ (Triticum estivum) Wheat } & \multirow[t]{3}{*}{ Shahbaz banda } & Rhizopus. spp & 60 & 24 \\
\hline & & Mucor. spp & 20 & 4 \\
\hline & & Ascomycetes & 20 & 4 \\
\hline & \multirow[t]{2}{*}{ Yarhussain } & Penicilium. spp & 40 & 12 \\
\hline & & Mucor.spp & 20 & 8 \\
\hline & \multirow{6}{*}{ Parhala } & A. alternata & 100 & 20 \\
\hline & & Erysiphe. spp & 80 & 12 \\
\hline & & Penicilium. spp & 60 & 12 \\
\hline & & Aspergillus niger & 40 & 8 \\
\hline & & A. alternata & 60 & 12 \\
\hline & & Erysiphe. spp & 80 & 32 \\
\hline & \multirow[t]{3}{*}{ Sudher } & Erysiphe. spp & 60 & 12 \\
\hline & & A. alternate & 60 & 32 \\
\hline & & Penicilium. spp & 80 & 16 \\
\hline & Nelor & Penicilium. spp & 60 & 32 \\
\hline & \multirow[t]{4}{*}{ Dagai } & Cladosporium & 20 & 4 \\
\hline & & A. alternata & 60 & 20 \\
\hline & & A. solani & 40 & 32 \\
\hline & & Rhizopus. spp & 20 & 4 \\
\hline & \multirow[t]{4}{*}{ Tordher } & F. solani & 60 & 32 \\
\hline & & A. alternate & 80 & 40 \\
\hline & & Penicilium. spp & 40 & 8 \\
\hline & & Cladoporium. spp & 40 & 16 \\
\hline & \multirow[t]{2}{*}{ Kernal Sher Khan } & Penicilium. spp & 80 & 40 \\
\hline & & Mucar.spp & 40 & 8 \\
\hline & \multirow[t]{2}{*}{ Dobian } & Penicilium. spp & 20 & 4 \\
\hline & & A. alternata & 60 & 16 \\
\hline & Marghuz & F. solani & 60 & 28 \\
\hline & & A. alternate & 80 & 24 \\
\hline & & Penicilium. spp & 40 & 8 \\
\hline & & Cladoporium, spp & 40 & 12 \\
\hline & & Erysiphe. spp & 40 & 20 \\
\hline & Maini & A. solani & 80 & 16 \\
\hline & & Penicilium. spp & 40 & 16 \\
\hline & & Erysiphe. spp & 20 & 4 \\
\hline & Darra & Erysiphe. spp & 80 & 16 \\
\hline & & A. solani & 40 & 8 \\
\hline
\end{tabular}




\begin{tabular}{|c|c|c|c|c|}
\hline & & Aspergillus. spp & 60 & 12 \\
\hline & \multirow[t]{2}{*}{ Kunda } & A. solani & 80 & 16 \\
\hline & & Penicilium & 60 & 12 \\
\hline & \multirow[t]{4}{*}{ Jalbai } & Erysiphe. spp & 40 & 8 \\
\hline & & A. solani & 60 & 4 \\
\hline & & Candida vulgaris & 20 & 4 \\
\hline & & Penicilium. spp & 40 & 8 \\
\hline & \multirow[t]{2}{*}{ Ghazi } & A. alternata & 60 & 4 \\
\hline & & R. solani & 60 & 28 \\
\hline \multirow[t]{18}{*}{ Allium sativum (Garlic) } & & Mucor.spp & 60 & 12 \\
\hline & & Aspergillus. spp & 40 & 84 \\
\hline & \multirow{3}{*}{ Darra } & F. solani & 60 & 20 \\
\hline & & A. solani & 80 & 36 \\
\hline & & Penicilium. spp & 40 & 16 \\
\hline & \multirow[t]{4}{*}{ Takhat Baye } & A. solani & 60 & 36 \\
\hline & & Rhizopus. spp & 40 & 16 \\
\hline & & Albugo candida & 20 & 8 \\
\hline & & F. solani & 40 & 16 \\
\hline & \multirow[t]{2}{*}{ Kunda } & A. solani & 25 & 16 \\
\hline & & Albugo candida & 50 & 20 \\
\hline & \multirow[t]{4}{*}{ Tordher } & Penicilium. spp & 40 & 8 \\
\hline & & A. solani & 80 & 52 \\
\hline & & Rhizopus & 60 & 28 \\
\hline & & F. solani & 60 & 20 \\
\hline & \multirow[t]{3}{*}{ Topi } & A. solani & 80 & 32 \\
\hline & & R. solani & 60 & 44 \\
\hline & & A. niger & 20 & 8 \\
\hline \multirow{14}{*}{$\begin{array}{l}\text { (Brassica compestress) } \\
\text { Mustard }\end{array}$} & \multirow[t]{3}{*}{ Dobian } & Rhizopus. spp & 80 & 16 \\
\hline & & Mucor. spp & 40 & 8 \\
\hline & & Penicilium. spp & 20 & 4 \\
\hline & \multirow[t]{2}{*}{ Kalu khan } & A. alternate & 80 & 38 \\
\hline & & Penicilium. spp & 20 & 8 \\
\hline & \multirow[t]{3}{*}{ Takht Baye } & A. solani & 40 & 8 \\
\hline & & M. phaseolina & 60 & 12 \\
\hline & & B. cineria & 40 & 8 \\
\hline & \multirow[t]{2}{*}{ Shabaz gallei } & Geotricum candidum & 60 & 18 \\
\hline & & Rhizopus. spp & 80 & 12 \\
\hline & \multirow[t]{2}{*}{ Bagh Banda } & A. solani & 60 & 16 \\
\hline & & Penicilium. spp & 20 & 4 \\
\hline & \multirow[t]{2}{*}{ Kunda } & Rhizopus. spp & 60 & 8 \\
\hline & & M. phaseolina & 60 & 20 \\
\hline \multirow[t]{15}{*}{ Trifolium repens Clover } & \multirow[t]{2}{*}{ Swabi } & A. alternata & 60 & 40 \\
\hline & & Penicilium. spp & 40 & 32 \\
\hline & Jalabai & M. phaseolina & 60 & 12 \\
\hline & & A. alternata & 40 & 24 \\
\hline & & Erysiphe. spp & 60 & 12 \\
\hline & & Penicilium. spp & 40 & 20 \\
\hline & Sudher & M. phaseolina & 80 & 24 \\
\hline & & A. alternata & 40 & 8 \\
\hline & & A. niger & 60 & 12 \\
\hline & & Penicilium. spp & 60 & 12 \\
\hline & Sheikh meltoon & A. flavus & 80 & 16 \\
\hline & & R. solani & 60 & 12 \\
\hline & & Cladosporium.spp & 40 & 8 \\
\hline & & A. alternata & 20 & 4 \\
\hline & & Erysiphe. spp & 020 & 44 \\
\hline (Allium cepa) Onion & Thorder & Erysiphe. spp & 60 & 24 \\
\hline
\end{tabular}




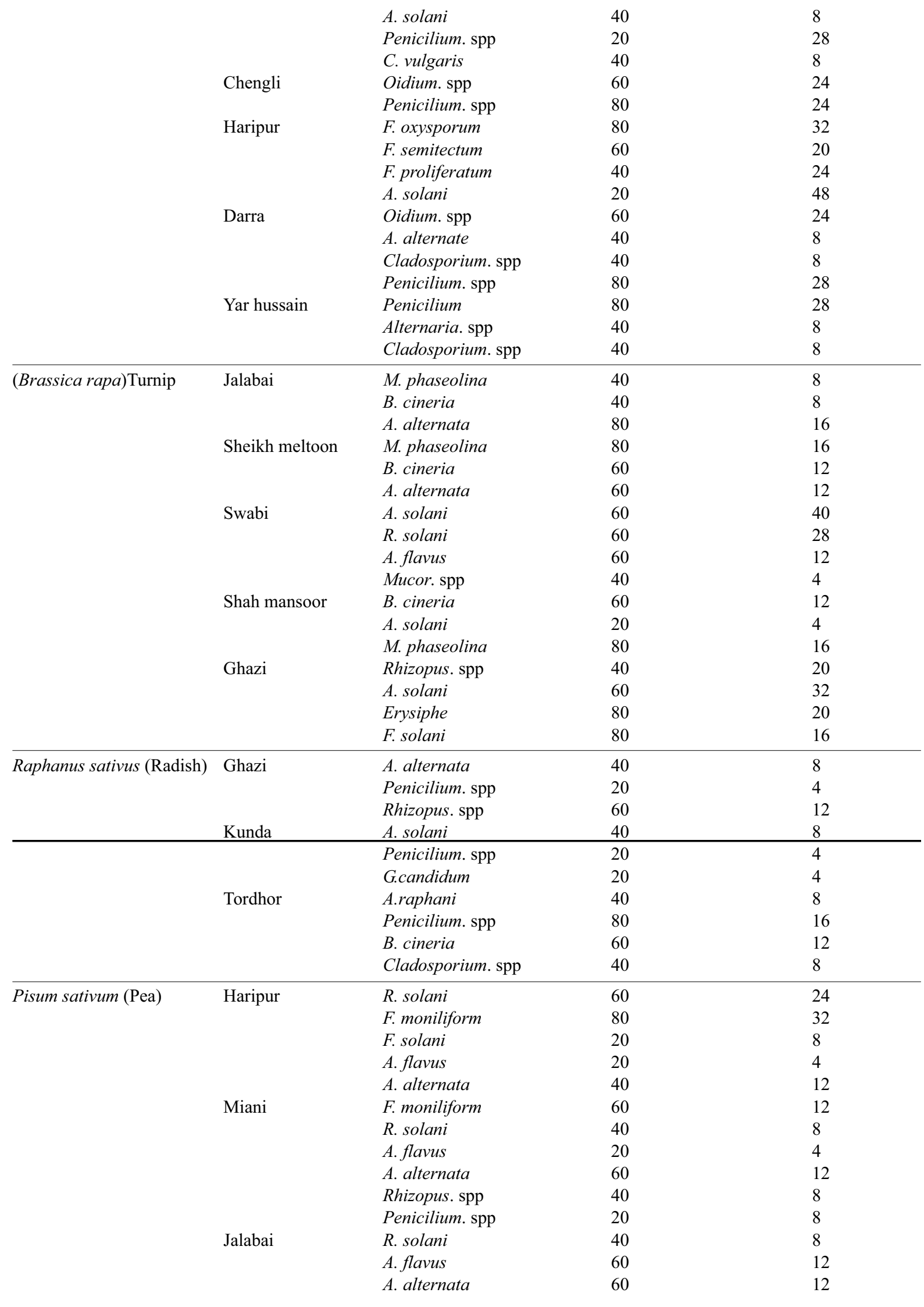



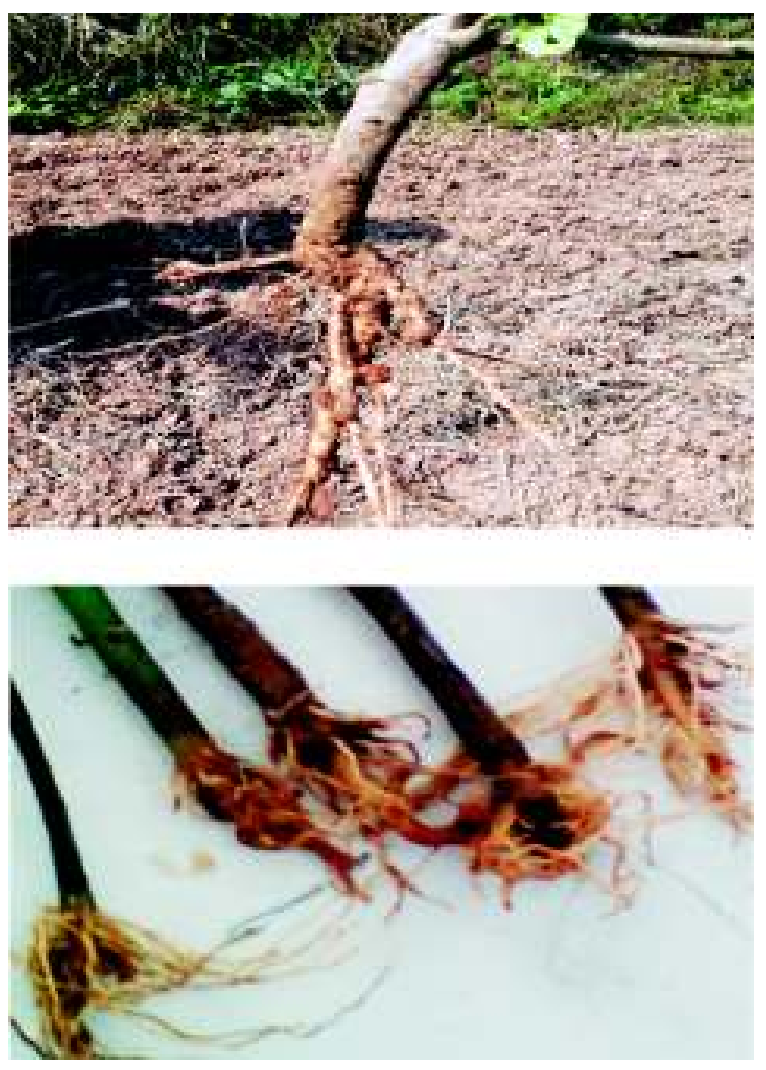

Fig. 1. Root-knot infection caused by Meloidogynae spp in Brinjal and Chili plant.

significant at $\mathrm{P}$-value $=0.01$. The radish was associated with different pathogens, while the infection percentage

Table 3. Pearson correlation of infection and colonization percentage of different economically importance of crops of Pakistan.

\begin{tabular}{llll}
\hline \hline $\begin{array}{l}\text { Scientific name of } \\
\text { crop }\end{array}$ & $\begin{array}{l}\text { Common } \\
\text { name }\end{array}$ & $\begin{array}{l}\text { Pearson } \\
\text { correlation }\end{array}$ & P-value \\
\hline Nicotiana tobacum & Tobacco & $0.924^{* *}$ & 0.001 \\
Abelmoscus esculantus & Ladyfinger & $0.765^{* * *}$ & 0.000 \\
Capsicum annum & Chili & $0.766^{* * *}$ & 0.000 \\
Solanum melongena & Eggplant & $0.78^{* * *}$ & 0.000 \\
Zea mayz & Maize & $0.977^{* *}$ & 0.004 \\
Solanum lycopersicum & Tomato & $0.779^{*}$ & 0.013 \\
Triticum estivum & Wheat & $0.614^{* * *}$ & 0.000 \\
Allium sativum & Garlic & 0.325 & 0.161 \\
Brassica compestres $)$ & Mustard & $0.737^{* *}$ & 0.003 \\
Trifolium repens & Clover & 0.335 & 0.223 \\
Allium cepa & Onion & $0.647^{* *}$ & 0.005 \\
Brassica rapa & Turnip & 0.377 & 0.136 \\
Raphanus sativus & Radish & $1.00^{* *}$ & 0.000 \\
Pisum sativum & Pea & $0.798^{* *}$ & 0.001 \\
\hline \hline
\end{tabular}

Note: $*=\mathrm{P}<0.05 ; * *=\mathrm{P}<0.01 ; * * *=\mathrm{P}<0.001$
Table 4. Comparison of means losses of different economically important crops of Pakistan.

\begin{tabular}{lllll}
\hline \hline Crop & Mean & $\begin{array}{l}\text { Std } \\
\text { deviation }\end{array}$ & $\mathrm{t}$ & $\begin{array}{l}\mathrm{P}- \\
\text { value }\end{array}$ \\
\hline Nicotiana tobacum & 40.667 & 17.93 & 3.929 & 0.059 \\
Abelmoscus & $58^{* * *}$ & 9.55 & 14.87 & 0.000 \\
Capsicum annum & $50.5 * * *$ & 13.95 & 10.24 & 0.000 \\
Solanum melongena & $47^{* * *}$ & 19.88 & 7.48 & 0.000 \\
Zea mayz & $61.33^{*}$ & 14.01 & 7.58 & 0.017 \\
Solanum & 45.5 & 27.31 & 2.89 & 0.102 \\
Lycopersicum & & & & \\
Triticum estivum & $28.75^{* * *}$ & 15.62 & 8.38 & 0.000 \\
Allium sativum & $22.42^{*}$ & 18.74 & 2.93 & 0.033 \\
Brassica & $28.29^{* * *}$ & 9.07 & 8.25 & 0.000 \\
compestres) & & & & \\
Trifolium repens & $33.25 * *$ & 11.03 & 6.03 & 0.009 \\
Allium cepa & $27.63^{* * *}$ & 9.84 & 7.94 & 0.000 \\
Brassica rapa & $46.5^{* *}$ & 21.92 & 5.97 & 0.003 \\
Raphanus sativus & $25^{*}$ & 4.36 & 9.93 & 0.01 \\
Pisum sativum & $40.88^{*}$ & 17.48 & 4.68 & 0.018 \\
\hline \hline
\end{tabular}

Note: $*=\mathrm{P}<0.05 ; * *=\mathrm{P}<0.01 ; * * *=\mathrm{P}<0.001$

of Rhizopus spp. was amaximum of $60 \%$ and responsible up to $30 \%$ losses in Thorder, while mean differences of losses (25) was found significant at $\mathrm{P}$-value $=0.05$. Maximum infection percentage of $F$. moniliform wasa maximum of $80 \%$ and Pearson correlation $(0.798)$ reveal significant at $\mathrm{P}$-value $=0.01$ between percent infection and colonization which was responsible 20 to $62.5 \%$ losses in Pea plant, while mean differences of losses (40.88) was found significant at $\mathrm{P}$-value $=0.01$. (Table 1- 4). The soil-borne pathogens have been received little attention which is caused the extent of losses (McDonald and Linde, 2002) and is very difficult to control (Haas and Defago, 2005). Fungi and root-knot nematodes attacking the roots of crop plants in Pakistan have been reported (Kayani and Mukhtar, 2018; Khan et al., 2017; Hussain et al., 2016). The most common root-rot pathogens found in all the visited areas in 14 different plant species were M. phaseolina, $R$. solani, A. alternate, A.solani and two species of Fusarium namely $F$. oxysporum and $F$. solani. While two species of root-knot nematode $M$. incognita and $M$. javanica were also found causing root knot disease.

In combination or alone these pathogens causing huge losses. Annual losses of over 10 million in tobacco $(N$. tabacum) from black shank have been reported in North Carolina alone (Mila and Radcliff, 2014). M. phaseolina, R.solani and F. solani were found responsible up to $52 \%$ in Seen Khel and 50\% in Gohati in the fields of 
tobacco plants. In ladyfinger (E. esculentus) maximum loss $(72 \%)$ was recorded in the area of Bamkhel caused by F. solani with the combination of other fungi. Chilli fields located in the area of Panjtar were severely infected with the combine effects of M. phaseolina and $M$. javanica and responsible to cause $65 \%$ losses. Jalaluddin et al. (2008) reported that charcoal root rot disease caused by Macrophomina phaseolina in sunflower (Helianthus annus), more than 500 different hosts can infect by this pathogen (Khan, 2007). In chili pepper plant pathogens attack roots, stems, leaves and fruits and cause $70 \%$ to $100 \%$ yield losses (Liu and LU, 2003).

Eggplant in the area of Nogram was severely infected with the combine effect of M. javanica and F. oxysporum and responsible to cause $76 \%$ losses. Effect of $F$. oxysporum and Meloidogyne are known to increase disease severity in cotton (Starr et al.,1989), other crops (Fateh et al., 2017). Mukhtar et al.(2017a) reported losses of crops due to plant-parasitic nematodes were 100 billion per year to world agriculture. The association of fungi with pathogenic nematodes caused greater losses has been reported (Rivera and Aballay, 2008). Vascular wilt fungus Fusarium spp. have been recognized for many years and the association of rootknot nematodes and severity of Fusarium wilt of cotton was greater when Meloidogyne incognita infected plants (Atikinson, 1892). In 34 districts of Punjab, Meloidogyne incognita was found with ubiquitous distribution (Irum, 2009; Khan and Ahmad, 2000). The common incidence of these root rot pathogens in Pakistan is presumably due to a temperature of $25-35{ }^{\circ} \mathrm{C}$ favorable for the growth of these pathogens which prevails in most of the year. A temperature of $26-30^{\circ} \mathrm{C}$ is optimum for the growth of $R$. solani $28-35{ }^{\circ} \mathrm{C}$ (Papavizas and Klag, 1970) for M. phaseolina (Dhingra and Sinclair, 1978) 25-30 ${ }^{\circ} \mathrm{C}$ for $F$. oxysporum and $27-30{ }^{\circ} \mathrm{C}$ for $F$. solani (Domsch et al.,1980), whereas, the root-knot nematodes (Meloidogyne spp.) are found to cause a 5\% loss on aworld-wide basis (Cetintas and Yarba, 2010).

Conflict of Interest. The authors declare no conflict of interest.

\section{References}

Akhtar, K.P,. Saleem, M. Y., Asghar,M., Haq, M.A. 2004. New report of Alternaria alternata causing leaf blight of tomato in Pakistan. Plant Pathology. 53.
Aslam, M.N., Mukhtar, T., Ashfaq, M., Hussain, M.A. 2017a. Evaluation of chili germplasm for resistance to bacterial wilt caused by Ralstonia solanacearum. Australas. Plant Pathology, 46: 289-292.

Aslam, M.N., Mukhtar, T., Hussain, M.A., Raheel, M. 2017b. Assessment of resistance to bacterial wilt incited by Ralstonia solanacearum in tomato germplasm. Journal of Plant Disease and Protection, 124: 585-590.

Atikinson, G.F. 1892. Some disease of cotton Alabama Polytechnical Institute of Agriculture. Experimental Station Bulletin, 41: 61-65.

Avelino, J., Cristancho, M., Georgiou, S., Imbach, P., Aguilar, L., Bornemann, G. 2015. The coffee rust crises in Colombia and Central America (2008-2013): impacts, plausible causes and proposed solutions. Food Security, 7: 303-21

Bajwa, R., Mukhtar,I., Mushtaq. S. 2010. New report of Alternaria alternata causing leaf spot of Aloe vera in Pakistan. Canadian Journal of Plant Pathology, 32: 490-492.

Baloch, G.N., Samrah, T., Syed Ehteshamul-Haque, Mohammad, A., Viqar, S., Jehan, A. 2013. Management of root diseases of eggplant and watermelon with the application of Asafoetida and seaweeds. Journal of Applied Botany and Food Quality, 86: 138-142.

Chehri, K., Abbasi, S., Redd, K.R.N., Salleh, B. 2010. Occurrence and pathogenicity of various pathogenic fungi on cucurbits from Kermanshah province, Iran. African Journal of Microbiology Research, 4: 1215-1223.

Cetintas, R., Yarba, M.M. 2010. Nematicidal effects of five essential plant oils on the southern root-knot nematode, Meloidogyne incognita race 2. Journal of Animal and Veterinary Advances, 9: 222-225.

Das, I.K., Fakrudin, B., Arora, D.K. 2008. RAPD cluster analysis and chlorate sensitivity of some Indian isolates of Macrophomina phaseolina from sorghum and their relationships with pathogenicity. MicroBiological Research. 163: 215-224.

De Groote, H., Dema, G., Sonda, G.B., Gitonga, Z.M. 2013. Maize for food and feed in east Africa-The farmers' perspective. Field Crops Research, 153: 22-36.

Dhingra, O.D., Sinclair, J.B. 1978. Biology and Pathology of Macrophomina phaseolina. Impress Universitaria, Universidade Federal Devicosa. Vicosa-Minas Gerais, Brazil, 166.

Domsch, K.H., Gams,W., Anderson, T.H., 1980. 
Compendium of Soil Fungi. 1, pp 858, Academic Press New York, USA.

Fateh, F.S., Mukhtar, T., Kazmi, M.R., Abbassi, N.A., Arif, A.M. 2017. Prevalence of citrus decline in district Sargodha. Pakistan Journal of Agricultural Science, 54: 9-13.

Egal, S., Hounsa, A., Gong, Y.Y., Turner, P.C., Wild, C.P., Hall, A.J., Hell, K., Cardwell, K.F. 2005. Dietary exposure to aflatoxin from maize and groundnut in young children from Benin and Togo, west Africa. International Journal of Food Microbiology, 104: 215-224.

Haas, D., Defago, G. 2005. Biological control of soilborne pathogens by fluorescent pseudomonads. Nature Reviews Microbiology, 3: 307-319

Hussain, M.A., Mukhtar, T., Kayani, M.Z. 2016. Reproduction of Meloidogyne incognita on resistant and susceptible Okra cultivars. Pakistan Journal of Agricultural Science, 53: 371-375.

Hussain, M.A., Mukhtar, T., Kayani, M.Z. 2011a. Assessment of the damage caused by Meloidogyne incognita on Okra. Journal of Animal and Plant Science, 21: 857-861.

Hussain, M.A., Mukhtar, T., Kayani, M.Z., Aslam M.N., Haque, M.I. 2012. A survey of Okra (Abelmoschus esculentus) in the Punjab province of Pakistan for the determination of prevalence, incidence and severity of root-knot disease caused by Meloidogyne spp. Pakistan Journal of Botany, 44: 2071-2075.

Islam, S., Samiul, H., Islam, M.H., Emdad, E.M., Halim, A., Hossen, Q.M. 2012. Tools to kill: genome of one of the most destructive plant pathogenic fungi Macrophomina phaseolina. BMC Genomics, 13: 493.

Inam-ul-haq., Mehmood, S., Rehman, H.M., Ali, Z., Tahir, M.I. 2012. Incidence of root rot diseases of soybean in Multan Pakistan and its management by the use of plant growth promoting rhizobacteria. Pakistan Journal of Botany, 44: 2077-2080.

Irum, M. 2009. Sunflower disease and insect. Pest in Pakistan: A review. African Crop Science Journal, 17: 109-118.

Jalaluddin, M., Hamid, M., Muhammad, S.E. 2008. Selection and application of a vam-fungus for promoting growth and resistance to charcoal rot disease of sunflower var. Helicon-250. Pakistan Journal of Botany, 40: 1313-1318.

Katan, J. 2017. Diseases caused by soilborne pathogens: biology, management and challenges. Journal of
Plant Pathology, 99: 305-315

Kayani, M.Z., Mukhtar, T., Hussain, M.A. 2018. Interaction between nematode inoculum density and plant age on growth and yield of cucumber and reproduction of Meloidogyne incognita. Pakistan Journal of Zoology, 50: 897-902.

Khan, H., Ahmad, R. 2000. Geographical distribution and frequency of occurrence of root-knot nematodes in Punjab-Pakistan. International journal of Agriculture and Biology, 2: 111-118.

Khan, A.R., Javed, N., Sahi, S.T.,Mukhtar, T., Khan, S.A., Ashraf, W. 2017. Glomus mosseae (Gerd \& Trappe) and neemex reduce invasion and development of Meloidogyne incognita. Pakistan Journal of Zoology, 49: 841-847.

Liu, R.Z., Lu, J. 2003. Inhibition of Trichoderma harzianum against the soil born fungal diseases of Capsicum. Journal of Zhongkai Agrotech College, 16: 6-11.

McDonald, B.A., Linde, C. 2002. Pathogen population genetics, evolutionary potential, and durable resistance. Annual Review of Phytopathology, 40: 349-379.

Mila, A.L., Radcliff, J. 2014. Managing diseases. In: Flue-Cured Tobacco-Guide. N.C. Copp. Ext. Serr. Bull, pp. 124-156. North Carolina State University, Raleigh,

Mohsan, M.M., Intizar-ul-Hassan., Liaquat, A. 2011. Chemotheraptic management of Alternaria black spot (Alternaria alternata) in mango fruits. Journal of Agricultural Research, 49: 499-505

Mukhtar, T., Hussain, M.A., Kayani, M.Z. 2017a. Yield responses of 12 Okra cultivars to southern rootknot nematode (Meloidogyne incognita). Bragantia, 75: $108-112$.

Nowicki, M., Nowakowska, M., Niezgoda,A., Kozik, E.U. 2012. Alternaria black spot of crucifers: symptoms, importance of disease and perspectives of resistance breeding. Vegetable Crops Research Bulletin, 76: 5-19.

Papavizas, G.C., Klag, N.G. 1970. Colonization and Growth of Rhizoctonia solani in Soil. In: Rhizoctonia solani. Biology and Pathology. (Ed.): Parmeter, J.R., 108-122, University of California Press, Berkeley, Loss Angeles and London.

Parveen, G., Urooj, F., Moin, S., Farhat, H., Fahim, M.F.S., Ehteshamul-Haque. 2020. Estimation of lossess caused by root totting fungi anf root knot nematodes infecting some important crops in lower Sindh and Hub, Balochistan of Pakistan. Pakistan 
Journal of Botany, 52: DOI: http://dx.doi.org/10. 30848/PJB2020-2(15).

Rehman, A., Laun, J.D., Shahzad, B., Chandio, A.A., Hussain, I., Nabi, G., Iqbal, M.S. 2015. Economic perspectives of major field crops of Pakistan: An empirical study. Science Directory, 1: 145-158.

Rivera, L., Aballay, E. 2008. Nematicidal effect of various organic soil amendments on Meloidogyne ethiopica white head, (1968), on potted vine plants. Chilean Journal of Agricultural Research, 68: 290296.

Savary, S., Ficke, J.A., Aubertot, N., Hollier, C. 2012. Crop losses due to diseases and their implications for global food production losses and food security. Food Secience, 4: 519-537.

Shafique, H.A., Sultana, V., Ehteshamul-Haque, S., Athar, M. 2016. Management of soil-borne diseases of organic vegetables. Journal of Plant Protection Research, 56: 221-230.

Sokal, R.R., Rohlf, R.F. 1995. Biometry: the Principles and Practice of Statistics in Biological Research, $2^{\text {nd }} E d$. Freeman Publishers.DOI: 10.2307/2343822.

Starr, J.L., Jeger, M.J., Marlyn, R.D., Schilling, K. 1989. Effects of Meloidogyne incognita and Fusarium oxysporum F. spp. vasinfectum on plant mortality and yield of cotton. Phytopathology, 79: 640-646.

Suleiman, R., Rosentrater, K., Bern,C. 2013. Effects of deterioration parameters on storage of maize: a review. Journal of Natural Sciences Research, 3: 147-165.

Suleiman, R.A., Kurt, A., Rosentrater. 2015. Current maize production, postharvest losses and the risk of mycotoxins contamination in Tanzania. Agricultural and Biosystems Engineering Conference Proceedings and Presentations, IOWA state University.

Tariq-Khan, M., Munir, A., Mukhtar, T., Hallmann, J., Heuer, H. 2017. Distribution of root-knot nematode species and their virulence on vegetables in northern temperate agro-ecosystems of the Pakistaniadministered territories of Azad Jammu and Kashmir. Journal of Plant Disease Protection, 124: 201-212

Taylor, A.L., Sasser, J.N. 1978. Biology, Identification of Root Knot Nematodes (Meloidogyne species). pp 111, North Carolina State University, Raleigh, Graphics, USA.

Weinberg, Z.G., Yan, Y., Chen, Y., Finkelman, S., Ashbell, G., Navarro, S. 2008. The effect of moisture level on high-moisture maize (Zea mays L.) under hermetic storage conditions-in vitro studies. Journal of Stored Products Research, 44: 136-*144

Zarina, B., Shahina, F. 2010. Research work carried out on the management of root knot nematode diseases in Pakistan. Pakistan Journal of Nematology, 28: 153-239. 